\begin{tabular}{|c|l|}
\hline Title & Fabrication of stable Pd nanowire assisted by hydrogen in solution \\
\hline Author(s) & Kiguchi, M.; Murakoshi, K. \\
\hline Citation & $\begin{array}{l}\text { A pplied Physics Letters, 88(25), 253112 } \\
\text { https://doi.org/10.1063/1.2216029 }\end{array}$ \\
\hline Issue Date & 2006-06-19 \\
\hline Doc URL & http://hdl.handle.net/2115/14463 \\
\hline Rights & Copyright (c) 2006 A merican Institute of Physics \\
\hline Type & article \\
\hline File Information & GetPDFServlet.pdf \\
\hline
\end{tabular}

Instructions for use 


\title{
Fabrication of stable Pd nanowire assisted by hydrogen in solution
}

\author{
Manabu Kiguchi and Kei Murakoshia) \\ Department of Chemistry, Graduate School of Science, Hokkaido University, Sapporo 060-0810, Japan
}

(Received 9 February 2006; accepted 22 May 2006; published online 23 June 2006)

\begin{abstract}
We have mechanically fabricated a Pd nanowire in solution under electrochemical potential control. A clear feature appeared in the conductance histogram when the electrochemical potential of the Pd wire was kept at the hydrogen evolution potential. Conductance traces showed the Pd wire was stretched $0.4 \mathrm{~nm}$ in length just before breaking, suggesting that at least two $\mathrm{Pd}$ atoms might contribute to the formation of the $\mathrm{Pd}$ wire. The results indicate that a certain atomic configuration of the Pd nanowire is stabilized by hydrogen. We discuss the stabilization mechanism due to changes in bond strengths caused by hydrogen adsorption or incorporation. (C) 2006 American Institute of Physics. [DOI: 10.1063/1.2216029]
\end{abstract}

Fabrication and characterization of metal atomic wires have attracted attention due to their potential applications in future electronic devices. ${ }^{1}$ Recently, Ir, Pt, and Au monoatomic wires have been fabricated by pulling nanocontacts using a scanning tunneling microscope (STM) or a mechanically controllable break junction (MCBJ) in ultrahigh vacuum (UHV) at low temperatures. ${ }^{1-3}$ In addition to methods in UHV, the electrochemical method has been recognized as another powerful approach in the fabrication of metal nanowires. ${ }^{4-8}$ Recently, we have demonstrated that Fe, $\mathrm{Co}$, and $\mathrm{Ni}$ metal nanowires were stabilized in solution at room temperature via the hydrogen evolution reaction. 6,7 Furthermore, we observed results suggesting the formation of a monoatomic $\mathrm{Ni}$ wire in solution, while monoatomic wires of $3 d$ and $4 d$ metals have not been prepared in UHV up to now.

In the present study, we have studied the hydrogen assisted stabilization of the $\mathrm{Pd}(4 d$ metal) nanowire in the fabrication of a monoatomic wire. A monoatomic $\mathrm{Pd}$ wire is predicted to be ferromagnetic, ${ }^{9}$ and to test this hypothesis it is useful to fabricate this wire. In addition to the stabilization of the Pd nanowire by hydrogen, the interaction between hydrogen and the Pd nanowire should be interesting. Since the interaction between hydrogen and $\mathrm{Pd}$ is large, the nanowire of the palladium hydride formed in solution should have electrical properties. ${ }^{10}$

In our study of the $\mathrm{Pd}$ nanowire, we have chosen to measure the conductance. Electrical conductance through a metal nanowire on an atomic scale is expressed by $G$ $=2 e^{2} / h \sum T_{i}$, where $T_{i}$ is the transmission probability of the $i$ th conductance channel, $e$ is the electron charge, and $h$ is Planck's constant. ${ }^{1}$ Since conductance quantization depends on the atomic structure of a nanowire and the inherent properties of metals, we can study the atomic and electronic structures of metal nanowires by the conductance measurements. We have already studied conductance quantization of a Pd nanowire in solution. ${ }^{8}$ The conductance histogram displayed a clear feature peak at $1 G_{0}$, while conductance quantization was not observed in UHV at room temperature. ${ }^{11}$ At low temperatures in UHV, the appearance of the $1 G_{0}$ and $0.5 G_{0}$ features was reported in the conductance histogram for $\mathrm{Pd}$ under hydrogen dosing. ${ }^{12}$ At the present stage, it is clear

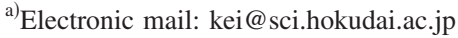

that hydrogen stabilizes the structure with a $1 G_{0}$ or $0.5 G_{0}$ in both UHV and solution. However, there has been little discussion about the structure, that is, it is not clear whether the conductance originates from the bridging hydrogen molecules or from the Pd nanostructure stabilized by hydrogen.

In the present study, we have investigated the mechanical properties of the Pd nanowire under electrochemical potential control. To obtain information about the structure of the nanowire, we have analyzed each conductance trace, as the conductance traces provide information about the nanowire such as wire length and thickness. Using the trace analysis results, we discuss the formation mechanism of the $\mathrm{Pd}$ monoatomic wire in solution.

The experimental design used in this study was the same as described in our previous report. ${ }^{5-8}$ An electrochemical scanning tunneling microscope (EC-STM) was used to fabricate the $\mathrm{Pd}$ nanowire. The electrochemical potential $\left(\Phi_{0}\right)$ was controlled using a potentiostat (Pico-Stat, Molecular Imaging Co.) with a $\mathrm{Ag} / \mathrm{AgCl}$ reference electrode. The electrolyte consisted of $1 \mathrm{mM} \mathrm{PdCl}, 2 \mathrm{mM} \mathrm{HCl}$, and $0.05 \mathrm{M}$ $\mathrm{H}_{2} \mathrm{SO}_{4}$. The Pd nanowire was prepared as follows. First, the electrochemical potentials of both STM tip and substrate were maintained at less than $+450 \mathrm{mV}$ where Pd bulk deposition proceeds. ${ }^{13}$ After the sufficient deposition of $\mathrm{Pd}$ onto the surfaces of the STM tip and the substrate, the tip was pressed into the substrate and contacts were then pulled out. During contact breaking, a Pd nanowire is formed between the tip and substrate. Conductance was measured during the breaking process under an applied bias of $20 \mathrm{mV}$ between the tip and substrate. Statistical data (conductance histograms, etc.) were obtained for a large number (over 3000) of individual conductance traces.

Figure 1(a) shows the cyclic voltammogram of the $\mathrm{Au}$ electrode in solution. Pd deposition and hydrogen adsorption/evolution proceeded when the electrochemical potential of $\mathrm{Au}$ electrodes were maintained under more negative conditions than $\Phi_{0}=450 \mathrm{mV}$ and $\Phi_{0}=-200 \mathrm{mV}$, respectively. ${ }^{13-15}$ Figures $1(\mathrm{~b})$ and 1 (c) show the conductance histogram of the $\mathrm{Pd}$ nanowire at $\Phi_{0}=-250 \mathrm{mV}$ and $\Phi_{0}$ $=100 \mathrm{mV}$, respectively. There is no clear feature in the conductance histogram at $\Phi_{0}=100 \mathrm{mV}$, suggesting that no preferential atomic configurations were formed. The featureless conductance histogram agreed with the previous results in UHV at room temperature. ${ }^{11}$ When the electrochemical potential of the nanowire was maintained under more negative 

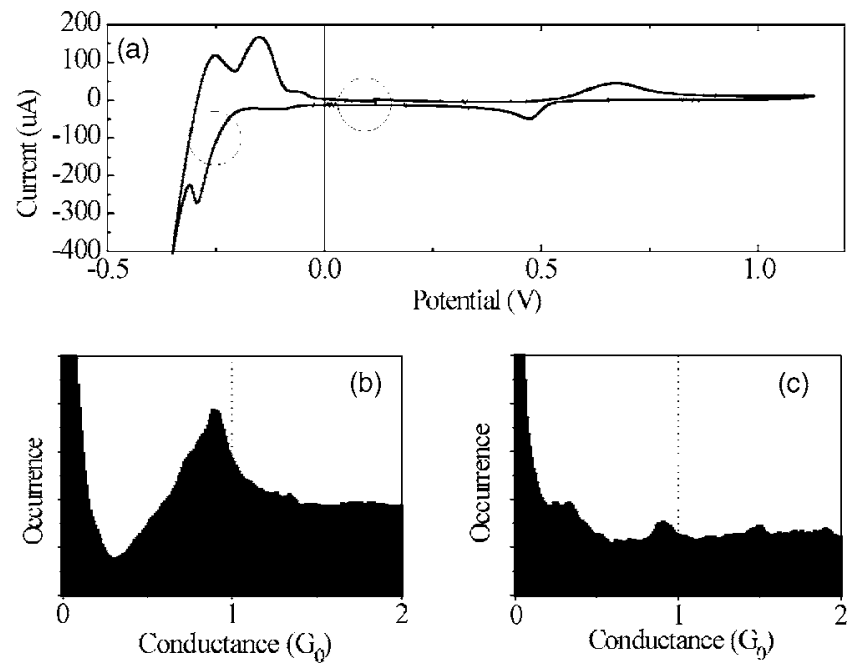

FIG. 1. (a) Cyclic voltammogram observed in $1 \mathrm{mM} \mathrm{PdCl}_{4}, 2 \mathrm{mM} \mathrm{HCl}$, and $0.05 \mathrm{M} \mathrm{H}_{2} \mathrm{SO}_{4}$. The conductance histograms at (b) $\Phi_{0}=-250 \mathrm{mV}$ and (c) $\Phi_{0}=100 \mathrm{mV}$.

conditions $\left(\Phi_{0}=-200 \mathrm{mV}\right)$, a feature appeared near the unit of the quantum conductance $\left(\sim 0.9 G_{0}\right)$. These results agree with our previous results. ${ }^{8}$

Next, we analyzed the conductance traces to determine the structure of the Pd nanowire. Figure 2 shows the typical conductance traces at (a) $\Phi_{0}=100 \mathrm{mV}$ and $(\mathrm{b}, \mathrm{c}) \Phi_{0}=$ $-250 \mathrm{mV}$. As a general tendency, the conductance changed in a stepwise fashion rather than continuously. At $\Phi_{0}$ $=100 \mathrm{mV}$, the conductance values of the plateaus were nonreproducible, resulting in featureless conductance histograms [Fig. 1(c)]. On the other hand, the $(0.6-1.2) G_{0}$ plateau with a stretched length ca. $0.5 \mathrm{~nm}$ appeared in the trace at $\Phi_{0}=$ $-250 \mathrm{mV}$, as shown in Fig. 2(b). These characteristic plateaus were the origin of the feature at $0.9 G_{0}$ in the conductance histogram [Fig. 1(b)]. The value of $0.9 G_{0}$ may correspond to that of a Pd monoatomic contact. This value is comparable to previously documented estimations of clean transition metal monoatomic contacts in UHV systems. ${ }^{1}$ The length of the plateau also showed dependence on the electrochemical potential. To evaluate characteristics of the plateau length quantitatively, we examined the distribution of lengths for the last conductance plateau. For comparison, we examined the Pt nanowire under the hydrogen evolution, in which a hydrogen molecule would bridge between Pt electrodes. Smit et al. showed that the $1 G_{0}$ feature appeared in the con-

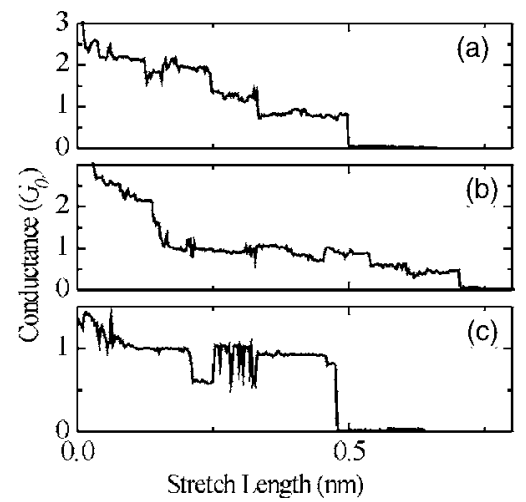

FIG. 2. Typical conductance traces of Pd nanowire at (a) $\Phi_{0}=100 \mathrm{mV}$ and [(b) and (c)] $\Phi_{0}=-250 \mathrm{mV}$.

ductance histogram under hydrogen dosing at low temperatures in UHV. ${ }^{16}$ By measuring the conductance and vibration spectra, they showed that the $1 G_{0}$ feature originated from a single hydrogen molecule bridging $\mathrm{Pt}$ electrodes. In the breaking trace, the $1 G_{0}$ plateau broke within $0.2 \mathrm{~nm}$, which is consistent with the small size of the hydrogen molecule. ${ }^{16}$ The inset of Fig. 3(a) shows the conductance histogram of the Pt nanowire under hydrogen evolution reaction in solution. A feature close to $1 G_{0}$ appears in the histogram. The feature appears to have originated from the bridging hydrogen molecule, although the situation in solution might be different from that in UHV at low temperatures. Figure 3(a) shows the distribution of lengths for the last conductance plateau for Pt. The length of the last plateau was defined as the distance between the points at which the conductance dropped below $1.3 G_{0}$ and $0.7 G_{0}$, respectively. These values were fixed in the following analysis. The contact broke at a short length of $\sim 0.2 \mathrm{~nm}$ which is in agreement with previous results. ${ }^{16}$

Figure 3(b) shows the distribution of lengths for the last conductance plateau for Pd at $\Phi_{0}=-250 \mathrm{mV}$. For comparison, we also show the distribution of the lengths for Pd at $\Phi_{0}=100 \mathrm{mV}$. While the contact broke within $0.2 \mathrm{~nm}$ at $\Phi_{0}$ $=100 \mathrm{mV}$, the plateau was stretched $0.4 \mathrm{~nm}$ in length at $\Phi_{0}=-250 \mathrm{mV}$. If a hydrogen molecule bridges between the Pd nanowire, the plateau length would be restricted to be $0.2 \mathrm{~nm}$ using the Pt results. The long plateau suggests that the $1 G_{0}$ feature did not originate from the hydrogen bridges. Since the Pd-Pd distance is $0.27 \mathrm{~nm}$ for bulk Pd, the $0.4 \mathrm{~nm}$ long $1 G_{0}$ plateau corresponds to a monoatomic wire of about

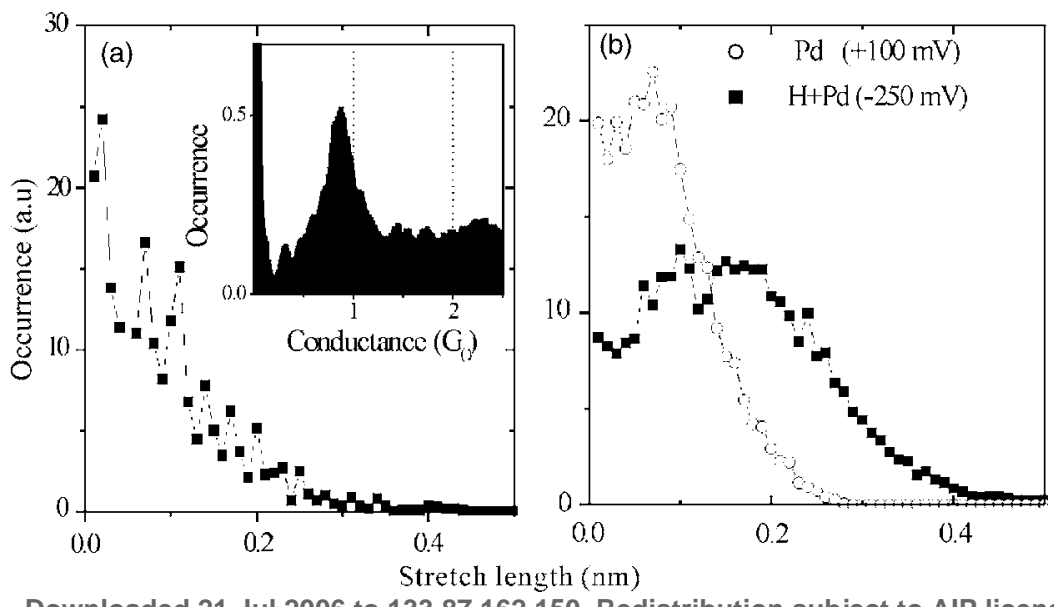

FIG. 3. (a) The distribution of lengths for the last conductance plateau for Pt under hydrogen evolution reaction $\left(\Phi_{0}=-800 \mathrm{mV}\right)$. Inset: Conductance histogram of Pt nanowire at $\Phi_{0}=-800 \mathrm{mV}$. (b) Distribution of lengths for the last conductance plateau for $\mathrm{Pd}$ at $\Phi_{0}$ $=100 \mathrm{mV}$ and $\Phi_{0}=-250 \mathrm{mV}$. 
two Pd atoms. A hydrogen atom would adsorb on the surface or be incorporated in the wire under the hydrogen evolution reaction, and thus, a stable Pd nanowire would be formed in solution.

The formation of a Pd monoatomic wire was also supported by the conductance trace. We occasionally observed reversible transition of conductance between a $1 G_{0}$ and $0.6-0.7 G_{0}$ as seen Fig. 2(c). A similar reversible transition of conductance was reported for $\mathrm{Au}$ monoatomic wires in the presence of physically adsorbed hydrogen in UHV at low temperatures $(10-30 \mathrm{~K}){ }^{17,18}$ Similar behavior was also observed for the Au monoatomic wire in solution under electrochemical potential control. ${ }^{19}$ The fractional conductance and reversible transition are explained by the formation of a dimerized structure in the Au monoatomic wire, and by the dynamic structural transition between a dimerized wire and an equal-spacing wire, respectively. The dimerization is a characteristic of a monoatomic wire. Therefore, the reversible transition of the conductance observed in the present study may reflect a similar dynamic structural transition in the Pd monoatomic wire. Present observations strongly suggest that the Pd monoatomic wire was fabricated in solution at room temperature under the hydrogen evolution reaction. Hydrogen stabilized the specific structure of the Pd monoatomic wire in solution at room temperature, leading to the characteristic conductance quantization behavior which has not observed in UHV at room temperature. At the present stage, it remains unclear whether hydrogen atom adsorbs on the wire or incorporates in the wire. In situ point contact spectroscopy should be indispensable to obtain an information on the structure. ${ }^{16}$

Adsorbed or incorporated hydrogen may play a decisive role in improving the stability of Pd monoatomic wire in solution. Generally, the bond strength between atoms increases when the coordination number of neighboring atoms becomes low. ${ }^{3}$ Further increments in the stabilization becomes significant in $5 d$ metals due to the relative effects of the valence electron, leading to the formation of a very stable nanowire of $5 d$ metals. In the case of $3 d$ and $4 d$ metals, it is known that stabilization of the bond strength due to changes in the coordination number as well as from the relative effect of electrons is not significant. These characteristics are key in the difficulty of preparing $3 d$ and $4 d$ metal nanowires showing stable conductance quantization. However, hydrogen adsorption/incorporation in the Pd nanowire is expected to change this situation. Adsorption of hydrogen to Pd proceeds via electron transfer from Pd to hydrogen. ${ }^{20}$ Since the top of the $4 d$ band of bulk Pd consists of states with antibonding characteristics, a decrease in the occupancy of the antibonding $4 d$ band due to hydrogen adsorption would increase the bond strength between Pd atoms. Thus, hydrogen adsorption/ incorporation may result in the stabilization of the Pd nanowire. This change in the electronic structure of the Pd nanowire under the hydrogen evolution reaction resulted in the fabrication of the stable Pd nanowire with $0.9 G_{0}$. Significant changes in the conductance value from $1.8 G_{0}$ clean $\mathrm{Pd}$ to $0.9 G_{0}$ hydrogen adsorption/incorporation Pd may also originate from this change in the electronic structure of the Pd nanowire. Finally, we briefly compare the present results with that in UHV at low temperature. While the $1 G_{0}$ and $0.5 G_{0}$ features were reported in the conductance histogram for Pd in UHV at low temperature, ${ }^{12}$ the $0.5 G_{0}$ feature was not observed in the conductance histogram under hydrogen evolution reaction in solution. In UHV, a hydrogen molecule is introduced to the Pd nanowire. On the other hand, in solution under hydrogen evolution reaction, an atomic hydrogen is formed on the Pd surface as dominant intermediate of the reaction. ${ }^{21}$ The situation in solution, which is different from that in UHV at low temperature, may lead to the absence of the structure of $0.5 G_{0}$ peak in the histogram.

In conclusion, we have observed conductance quantization behavior of mechanically fabricated $\mathrm{Pd}$ nanowire under electrochemical potential control. Under the hydrogen evolution reaction, a peak close to a $1 G_{0}$ appeared in the conductance histogram, suggesting that a certain atomic arrangement was maintained in solution. Relatively long $0.4 \mathrm{~nm}$ stretch lengths and reversible conductance fluctuations during stretching suggest the formation of a one-dimensional Pd monoatomic wire in solution at room temperature.

This work was partially supported by a Grant-in-Aid for Scientific Research A (No. 16205026) and Grant-in-Aid for Scientific Research on Priority Areas (No. 17069001) from MEXT.

${ }^{1}$ N. Agrait, A. L. Yeyati, and J. M. van Ruitenbeek, Phys. Rep. 377, 81 (2003).

${ }^{2}$ S. R. Bahn and K. W. Jacobsen, Phys. Rev. Lett. 87, 266101 (2001).

${ }^{3}$ R. H. M. Smit, C. Untiedt, A. I. Yanson, and J. M. van Ruitenbeek, Phys. Rev. Lett. 87, 266102 (2001).

${ }^{4}$ C. Shu, C. Z. Li, H. X. He, A. Bogozi, J. S. Bunch, and N. J. Tao, Phys. Rev. Lett. 84, 5196 (2000).

${ }^{5}$ J. Li, T. Kanzaki, K. Murakoshi, and Y. Nakato, Appl. Phys. Lett. 81, 123 (2002).

${ }^{6}$ M. Kiguchi, T. Konishi, and K. Murakoshi, Appl. Phys. Lett. 87, 043104 (2005).

${ }^{7}$ T. Konishi, M. Kiguchi, and K. Murakoshi, Jpn. J. Appl. Phys., Part 145 , 2000 (2006).

${ }^{8}$ J. Li, Y. Nakato, and K. Murakoshi, Chem. Lett. 34, 374 (2005).

${ }^{9}$ A. Delin, E. Tosatti, and R. Weht, Phys. Rev. Lett. 92, 057201 (2004).

${ }^{10}$ A. G. Lipson, B. F. Lyakhov, D. M. Sakov, and V. A. Kuznetsov, Phys. Solid State 38, 915 (1996).

${ }^{11}$ A. Enomoto, S. Kurokawa, and A. Sakai, Phys. Rev. B 65, 125410 (2002).

${ }^{12}$ Sz. Csonka, A. Halbritter, G. Milhaly, O. I. Shklyarevskii, S. Speller, and H. van Kepen, Phys. Rev. Lett. 93, 016802 (2004).

${ }^{13}$ H. Naohara, S. Ye, and K. Uosaki, J. Phys. Chem. B 102, 4366 (1998).

${ }^{14}$ S. Y. Liu, Y. H. Kao, Y. O. Sy, and T. P. Perng, J. Alloys Compd. 293-295, 468 (1999).

${ }^{15}$ A. Czerwinski, R. Matassi, and S. Zamponi, J. Electroanal. Chem. Interfacial Electrochem. 316, 211 (1991).

${ }^{16}$ R. H. M. Smit, Y. Noat, C. Untiedt, N. D. Lang, M. C. van Hemert, and J. M. van Ruitenbeek, Nature (London) 419, 906 (2002).

${ }^{17}$ Sz. Csonka, A. Halbritter, G. Mihaly, E. Jurdik, O. I. Shklyarevskii, S. Speller, and H. van Kempen, Phys. Rev. Lett. 90, 116803 (2003).

${ }^{18}$ Sz. Csonka, A. Halbritter, and G. Mihály, Phys. Rev. B 73, 075405 (2006).

${ }^{19}$ M. Kiguchi, T. Konishi, and K. Murakoshi, Phys. Rev. B 73, 125406 (2006).

${ }^{20}$ V. Ledentu, W. Dong, P. Sautet, G. Kresse, and J. Hafner, Phys. Rev. B 57, 12482 (1998).

${ }^{21}$ G. Jerkiewicz, Prog. Surf. Sci. 57, 137 (1998). 